\title{
How common is abuse in Greece? Studying cases with femoral fractures
}

\author{
I Trogan, N Dessypris, M Moustaki, E Petridou
}

\begin{abstract}
Aims-To examine the extent of undiagnosed child abuse in Greece by studying young children with femoral fractures, which may be associated with abuse.

Methods-Fifty seven consecutive cases of children under 6 years of age with femoral fracture were identified from the Emergency Department Injury Surveillance System database. Controls were 4162 children with other orthopaedic injuries from the same database.

Results-Whereas in the literature about one third of femoral injuries among young children are attributed to child abuse, no child in the studied series had been diagnosed or even investigated in this context. Nevertheless, the pattern of occurrence of femoral injuries was compatible with that of child abuse, in that patients were frequently very young boys of low socioeconomic status, and the accident had frequently occurred under poorly identified or implausible conditions at time periods when most family members were crowded at home.

Conclusions-Epidemiological risk factors for child abuse characterise femoral fractures in young children in Greece. It appears that child abuse is present in this country as in most other cultures. There is a clear need for refocusing medical personnel and hospital social services so that the problem is revealed, quantified, and appropriately dealt with. (Arch Dis Child 2001;85:289-292)
\end{abstract}

Keywords: child abuse; femoral fractures; injury

Child abuse is a major threat to the health of children throughout the world and occurs primarily in the family setting. The longer it goes undetected the more serious the consequences. In particular, repeated physical abuse of a child adversely affects their life in the family and in the community.

Although physical abuse is more easily identified than emotional or sexual abuse, documentation of the true magnitude of the problem is lagging behind in some societies, even with well functioning health and social services. ${ }^{2}$ In Greece, mild physical punishment of children is considered a normal aspect of child rearing. According to one study, $63 \%$ of mothers and $53 \%$ of fathers use some form of physical punishment in raising their children. ${ }^{3}$ It was stated that efforts to study the extent of child abuse "have met serious denial by both professionals and the public, as well as the state". Deeply rooted social values and attitudes about the protective role of the family inhibit the acceptance that the Greek family is as vulnerable to domestic violence as families elsewhere. Compared to other countries, the reported incidence of child abuse is minimal. ${ }^{3}$ It is possible, however, that this rate is an underestimate because of the lack of mandatory reporting of child abuse as well as inadequate awareness among health care workers. This makes it difficult to estimate the true extent of child abuse in Greece.

It has been reported that among children younger than 1 year, physical abuse is the leading cause of death and $63 \%$ of physically abused children are less than 2 years of age. ${ }^{4}$ Specific types of injuries such as bruises, subdural hematomas, or femoral fractures, however, are more frequently associated with abuse..$^{5-8}$ Data from the USA show that femoral fractures in children younger than 3 years of age, presenting to an emergency department, are highly suggestive of physical child abuse..$^{57}$ It is therefore reasonable to assume that the extent of recognition of young children presenting to emergency departments with femoral fractures as abuse victims could inversely reflect the level of underestimation of the child abuse problem by regional health services.

To examine the extent of undiagnosed child abuse in Greece, young children with femoral fractures presenting to emergency departments were re-evaluated for possible evidence of maltreatment. Data were retrieved from the national Emergency Department Injury Surveillance System (EDISS) run by the Center for Research and Prevention of Injuries. The study examined the risk profile of young children with femoral fractures by comparing the sociodemographic and event related characteristics of the case series to those of children presenting to the emergency department with orthopaedic injuries other than femoral fractures.

\section{Methods}

All children younger than 6 years of age with femoral fractures recorded by the EDISS during a two year period and unrelated to road traffic injuries were included. All injuries presenting to the emergency departments of four sentinel hospitals in Greece, two of which are located in the Greater Athens area, one in the county of Magnesia in mainland Greece, and one on the island of Corfu are recorded in the database. Specially trained research associates interview the guardian of the child on the basis of a precoded questionnaire; for those who are eventually admitted to hospital, 
Table 1 Demographic variables of the 57 children with femoral fracture and the 4162 same age other orthopaedic injury patients presenting to the participating emergency departments

\begin{tabular}{|c|c|c|c|c|}
\hline \multirow[b]{2}{*}{ Variable } & \multicolumn{2}{|c|}{ Femoral fracture } & \multicolumn{2}{|c|}{ Other orthopaedic injuries } \\
\hline & $n$ & $\%$ & n & $\%$ \\
\hline \multicolumn{5}{|l|}{ Age (mth) } \\
\hline$<12$ & 14 & 24.6 & 192 & 4.6 \\
\hline $12-23$ & 6 & 10.5 & 816 & 19.6 \\
\hline $24-35$ & 10 & 17.5 & 943 & 22.7 \\
\hline $36-47$ & 12 & 21.1 & 763 & 18.3 \\
\hline $48-59$ & 8 & 14.0 & 693 & 16.7 \\
\hline $60-71$ & 7 & 12.3 & 755 & 18.1 \\
\hline \multicolumn{5}{|l|}{ Gender } \\
\hline Boy & 37 & 64.9 & 2332 & 56.0 \\
\hline Girl & 20 & 35.1 & 1830 & 44.0 \\
\hline \multicolumn{5}{|l|}{ Nationality } \\
\hline Greek & 49 & 86.0 & 3895 & 93.6 \\
\hline $\begin{array}{l}\text { Country with GDP less } \\
\text { than that of Greece }\end{array}$ & 8 & 14.0 & 246 & 5.9 \\
\hline $\begin{array}{l}\text { Country with GDP higher } \\
\text { than that of Greece }\end{array}$ & 0 & 0.0 & 21 & 0.5 \\
\hline
\end{tabular}

Source: EDISS.

GDP, gross domestic product.

Table 2 Accident descriptive variables of the 57 children with femoral fracture and the 4162 same age other orthopaedic injury patients presenting to the participating emergency departments

\begin{tabular}{|c|c|c|c|c|}
\hline \multirow[b]{2}{*}{ Variable } & \multicolumn{2}{|c|}{ Femoral fracture } & \multicolumn{2}{|c|}{ Other orthopaedic injuries } \\
\hline & $n$ & $\%$ & $n$ & $\%$ \\
\hline \multicolumn{5}{|l|}{ Season } \\
\hline Winter & 8 & 14.1 & 831 & 20.0 \\
\hline Spring & 13 & 22.8 & 1117 & 26.8 \\
\hline Summer & 17 & 29.8 & 1109 & 26.6 \\
\hline Autumn & 19 & 33.3 & 1105 & 26.6 \\
\hline \multicolumn{5}{|l|}{ Day } \\
\hline Weekday & 33 & 57.9 & 2737 & 65.8 \\
\hline Weekend & 24 & 42.1 & 1425 & 34.2 \\
\hline \multicolumn{5}{|l|}{ Delay of seeking care } \\
\hline$\leqslant 2$ days & 53 & 93.0 & 3926 & 94.3 \\
\hline $3+$ days & 4 & 7.0 & 236 & 5.7 \\
\hline \multicolumn{5}{|l|}{ Time } \\
\hline Family at home (1600-2159) & 35 & 61.4 & 2107 & 50.6 \\
\hline $\begin{array}{l}\text { Sleeping or working } \\
(2200-1559)\end{array}$ & 22 & 38.6 & 2055 & 49.4 \\
\hline \multicolumn{5}{|l|}{ Mechanism of injury } \\
\hline Falls & 44 & 77.2 & 1663 & 40.0 \\
\hline $\begin{array}{l}\text { Struck accidentally by object or } \\
\text { person }\end{array}$ & 2 & 3.5 & 384 & 9.2 \\
\hline $\begin{array}{l}\text { Overexertion, strenuous } \\
\text { movements, etc }\end{array}$ & 11 & 19.3 & 2115 & 50.8 \\
\hline \multicolumn{5}{|l|}{ Place } \\
\hline Home & 33 & 57.9 & 3055 & 73.4 \\
\hline Other than home & 10 & 17.5 & 636 & 15.3 \\
\hline Inadequate information & 14 & 24.6 & 471 & 11.3 \\
\hline \multicolumn{5}{|l|}{ Number of injuries } \\
\hline 1 & 52 & 91.2 & 3980 & 95.5 \\
\hline 2 & 4 & 7.0 & 163 & 4.0 \\
\hline 3 & 1 & 1.8 & 19 & 0.5 \\
\hline \multicolumn{5}{|l|}{ Treatment } \\
\hline Treated and followed up & 16 & 28.1 & 4015 & 96.5 \\
\hline Hospitalised & 39 & 68.4 & 140 & 3.3 \\
\hline Refused admission to hospital & 2 & 3.5 & 7 & 0.2 \\
\hline
\end{tabular}

additional information is also collected. For the purpose of this study, medical records of all children with femoral fracture were selected from the database during the specified time period. The data were corroborated by personal interviews with treating physicians and the social services and studied for evidence of abuse. The figures of child abuse diagnoses among young children with femoral fractures were compared to previously publicised (mostly American) data.

All same age children with orthopaedic injuries caused by home and leisure accidents, comprised the vast majority of orthopaedic injuries recorded by the EDISS, and served as controls. Routinely collected data include age, gender, nationality, place of accident, time lapse between accident and presentation to the hospital, days spent in the hospital, type of treatment received, reported cause of injury at the time of admission, and referrals. The data were analysed through simple cross tabulations comparing children with femoral fractures to those with other orthopaedic injuries.

\section{Results}

A total of 57 children younger than 6 years of age with femoral fracture were identified from the 4219 children in the EDISS database who sustained home and leisure orthopaedic injuries during a two year period.

Table 1 shows the distribution of cases and controls by demographic characteristics. Of children with femoral fractures, almost one quarter were younger than 12 months whereas only $5 \%$ of children with other orthopaedic injuries belonged to this age group. While we had no other information concerning socioeconomic status for individual cases, femoral fractures were significantly more common among children whose parents originated from countries with low gross domestic product $(150 \%$ excess risk, $\mathrm{p}=0.003)$. There was also a small but not significant excess of boys among cases than controls. These findings are compatible with published information indicating that femoral fracture following child abuse is more common among male infants, and most frequently reported among those of low socioeconomic status. ${ }^{57}$

Table 2 shows the distribution of injured children (cases and controls) by conditions of injury and mode of treatment. There was a tendency for femoral fractures to occur during weekends and between 1600 and 2200, when most family members are at home, although these differences did not reach statistical significance. There was a non-significant deficit of cases of femoral fractures during the winter months, possibly because the seasonality of femoral fractures among young children does not resemble that of other orthopaedic accidents which are common during the winter. A small excess of cases among children for which care was sought after the passage of 48 hours was also not statistically significant.

On the contrary, there was a significant over representation of cases among those whose injury was attributed to "falls" and a suspiciously significant under representation of cases among those who were injured after an accidental strike by person or object $(p=0.001)$. Despite the fact that femoral fracture is a severe injury, a history of minor fall (fall on the same level or fall from height less than 1 metre) accounted for about half of femoral fractures but only one third of the control orthopaedic injuries (data not shown). Moreover, multiple injuries were more common-but not significantly differentamong cases than controls $(9.0 \% \quad v \quad 4.5 \%)$. There was also a revealing and significant excess of poor information concerning the place of accident among cases in comparison to controls $(p=0.005)$. Lastly, there was a tendency of more cases than controls to refuse admission to hospital. 
According to the discharge diagnosis on the computer database, no child was diagnosed as child abused, even though no child was identified to have a pathological bone condition that could explain the fracture. Fifty four of the 57 cases were determined to be accidental in nature. In three cases, the cause of the femoral fracture was classified as uncertain. Each of these cases had at least one factor that could raise the suspicion of abuse as all occurred at home from falls from height less than 1 metre. The case note review did not indicate that parents were asked whether they physically abused their child. In no case was the hospital social service notified, and there was no protocol in effect for declaring and reporting child abuse to the social service by paediatricians or auxiliary medical staff. Discussion with paediatric orthopaedic surgeons of the participating hospitals and a review of the official educational programme for orthopaedic fellows disclosed a total lack of formal training on the topic.

Based on data from the USA, ${ }^{57}$ as many as $30 \%$ of femoral fractures in children younger than 3 years may be attributable to child abuse; this percentage rises to $60 \%$ for infants younger than 1 year of age. Assuming that child abuse is as frequent in Greece as in the USA, 9/30 children younger than 3 years, included in this study, could have been victims of child abuse. Under the conservative assumption that child abuse is half as frequent as in the USA, 4-5 children of this age group might have been child abuse victims.

\section{Discussion}

This study, like most studies dealing with child abuse, faces a major obstacle: bias generated by denial is the rule rather than the exception. Thus, inferences can only be indirect and inherently uncertain. However, femoral fracture is as good an objective evidence as one could get, and data on their frequency and conditions of occurrence do allow some tentative conclusions. Helfer et al showed that a history of a minor fall that allegedly accounts for a femoral fracture should raise suspicion, because children less than 5 falling from height less than $90 \mathrm{~cm}$ have a minimal chance of suffering a fracture. ${ }^{9}$ In a study by Dalton et al, $31 \%$ of children less than 3 years of age presenting with femoral fractures were, eventually, diagnosed as child abused. ${ }^{7}$ Gross and Stranger stated that $50 \%$ of femoral fractures in children less than 5 years of age and $65 \%$ in children less than 1 year of age were attributed to child abuse. ${ }^{10}$ We decided to include children less than 6 years of age because we suspected that the abuse problem is not limited to infants, and femoral fractures among young mobile children can occur as an involuntary injury only under rare circumstances.

In our study, the profile of a young child with femoral fracture was compatible with that of a victim of child abuse. Thus, cases were more frequently than controls younger than 12 months, of male gender, and of low socioeconomic status. Although male gender and low socioeconomic status are also characteristics of involuntary injuries, the respective excesses were even more evident among injuries likely to reflect abusive behaviour. Moreover, the time of injury coincided with the time the whole family is crowded at home, and there was selective denial among cases of "striking" as possible cause, and substantial excess among them of vague stories describing the alleged accident. None of these observations is convincing in itself, but their coexistence creates a persuasive pattern. Lastly, parents of two children with femoral fracture, in addition to those who provided inadequate information, refused admission to hospital, a substantial proportional excess. Taking into account this pattern of occurrence and the evidence from studies in other countries, it is reasonable to conclude that between five and 15 children with femoral fractures were victims of abuse. These estimates rely on the fact that there were seven more children with femoral fracture ( $24 \%$ v 11\% expected) whose parents provided inadequate information, and two whose parents refused admission to hospital $(3.4 \% v$ $0.2 \%$ expected), over and beyond the excess numbers of femoral fractures occurring during weekends $(42.1 \% \quad v \quad 34.2 \%$ expected) and evening hours (61.4\% $v 50.7 \%$ expected). As in all studies based on stochastic processes it is not possible to identify which of the 57 children with femoral fractures were victims of child abuse, even though a likelihood value can be estimated for every child.

A number of studies have focused on child abuse, ${ }^{31-18}$ at least two of which were undertaken in Greece. ${ }^{318}$ However, our study has an advantage in that a control group has been used, facilitating the interpretability of the descriptive findings. For instance, many studies have pointed out that male infants and children are over represented among child abuse victims, but the observations need to be tempered by the fact that the male gender is over represented among victims of injuries of any type. Abusive behaviour may result in fractures in multiple sites. However, an epidemiological investigation is more powerful, when it focuses on the most discriminatory of these site specific fractures, in this case femoral fractures.

Our study indicates that although many of the children with femoral fracture had the profile of an abused child, none was formally characterised as such. Although we believe the underlying reason is collective denial in a society that considers itself child centred, several proximal factors should also be considered. During the review of the medical records of the patients, it became apparent that doctors had not indicated whether they specifically questioned parents about child abuse. Furthermore, parental descriptions of the alleged accident were not corroborated. This is unfortunate, because discrepant history is an important feature in the diagnosis of intentional trauma. ${ }^{19}$ Several paediatricians mentioned that they did not ask parents about child abuse because they were afraid of offending them. None recalled any training in child abuse during their medical studies. Sirotnak and Krugman believe that the recognition of any form of child abuse depends on the willingness 
of the physician to acknowledge its existence and to consider it in a differential diagnosis. ${ }^{19}$ What further complicates the issue is that in Greece there are no protocols or guidelines for reporting abuse once it is suspected. In this study, no child was referred to a child protection agency.

Agathonos et al outlined the profile of children who are predisposed to physical abuse in Greece, including variables such as offspring of unwanted pregnancies, male gender, low socioeconomic status, young parental age, social isolation, and poor relations with the family of origin. ${ }^{18}$ Physicians should take these factors into account, ask for details in the description of the event, and look carefully for physical signs of abuse such as marks of burns, bruises, and any history of previous fractures every time they are faced with a childhood injury. Moreover, an ad hoc child protection committee can assist hospital staff in identifying referral practices and provide collaboration in the management of suspected cases as well as teaching sessions for the staff. In some hospital settings, particularly in the USA, specific policies have been developed requiring that under special circumstances the hospital takes custody of the child pending notification of and intervention of the child protection services. ${ }^{20}$ Agathonos states that physical abuse may be considered as an exaggeration of socially accepted practices. ${ }^{3}$ Parental and well child visits provide another opportunity for physicians to undertake preventive efforts, and doctors should consult parents about pertinent rearing practices and discourage the use of physical discipline. Efforts should also be undertaken at the state level for modification of Greek laws in order to protect the welfare of children better. Given a law that requires only reasonable cause to suspect that a child had been abused or maltreated rather than certainty, there may be an increased number of false positive cases but the sensitivity of the measure will be substantially increased.

In conclusion, we have shown that femoral fracture and a set of epidemiological risk factors suggestive of child abuse are associated. Moreover, our data suggest that child abuse is present in Greece, as in most other cultures, and the issue is not whether the problem exists but how to measure and confront it.

We would like to thank the treating physicians of the participating hospitals and the Social Services Departments for their kind help.

1 Giardino AP, Christian CW, Giardino ER. A practical guide to the evaluation of child physical abuse and neglect. California: to the evaluation of child physi.

2 Besley MA. Child abuse: measuring a global problem. World Health Stat $Q$ 1993;46:69-73.

3 Agathonos-Georgopoulou H. Child maltreatment in Greece: a review of research. Child Abuse Review 1997;6: 257-71.

4 Waller AE, Baker SP, Szocka A. Child injury deaths: national analysis and geographic variations. Am $\mathcal{F}$ Public Health 1989;79:310-15.

5 Thomas SA, Rosenfield NS, Leventhal JM, Markowitz RI. Long-bone fractures in young children: distinguishing accidental injuries from child abuse. Pediatrics 1991;88:471-6.

6 McMahon P, Grossman W, Gaffney M, Stanitski C. Soft tissue injury as an indication of child abuse. F Bone foint Surg 1995;77:1179-83

7 Dalton HJ, Slovis T, Helfer RE, et al. Undiagnosed abuse in children younger than 3 years with femoral fracture. $A m \mathcal{F}$ Dis Child 1990;144:875-8.

8 Tzioumi D, Oates RK. Subdural hematomas in children under 2 years. Accidental or inflicted? A 10-year experience. Child Abuse Negl 1998;22:1105-12.

9 Helfer RE, Slovis TL, Black M. Injuries resulting when small children fall out of bed. Pediatrics 1977;60:533-5.

10 Gross RH, Stranger M. Causitive factors responsible for femoral fractures in infants and young children. 7 Pediatr Orthop 1983;3:341-4.

11 Ertem IO, Leventhal JM, Dobbs S. Intergenerational continuity of child physical abuse: how good is the evidence? Lancet 2000;356:814-19.

12 Socolar RR. Domestic violence and children. A review. $N C$ Med f 2000;61:279-83.

13 Glasser D. Child abuse and neglect and the brain-a review. f Child Psychol Psychiatry 2000;41:97-116.

14 Duncan AW. Radiological features of non- accidental injury. Hosp Med 1999;60:794-9.

15 Kocher MS, Kasser JR. Orthopaedic aspects of child abuse. 7 Am Acad Orthop Surg 2000;8:10-20.

16 Cahill LT, Kaminer RK, Johnson PG. Developmental, cognitive, and behavioural sequalae of child abuse. Child Adolesc Psychiatr Clin N Am 1999;8:827-43.

17 English DJ. The extent and consequences of child maltreatment. Future Child 1998;8:39-53.

18 Agathonos H, Stathacopoulou N, Adam H, Nakou S. Child abuse and neglect in Greece: sociomedical aspects. Child Abuse Negl 1982;6:307-11.

19 Sirotnak A, Krugman R. Physical abuse of children: an update. Pediatr Rev 1994;10:394-9.

20 University of Rochester Strong Memorial Hospital policy. Sect. 9.11.1 Suspected child abuse or maltreatment. Approved 14/6/93. 